\title{
matters \\ Pharmacokinetic profiles reconcile in vitro and in vivo activities of novel trypanocidal compounds
}

\author{
$\triangle$ Correspondence \\ pascal.maeser@swisstph.ch \\ 8 Disciplines \\ Parasitology \\ Q Keywords \\ Human African Trypanoso- \\ miasis \\ Trypanosoma Brucei \\ Differentiation \\ Pharmacokinetics \\ Chemotherapy \\ i- Type of Observation \\ Standalone \\ \& Type of Link \\ Negative data \\ (1) Submitted Jun 20, 2018 \\ (-) Published Aug 14, 2018
}

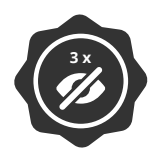

Triple Blind Peer Review The handling editor, the reviewers, and the authors are all blinded during the review process.

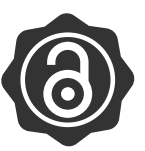

Full Open Access

Supported by the Velux Foundation, the University of Zurich, and the EPFL School of Life Sciences.

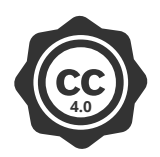

Creative Commons 4.0 This observation is distributed under the terms of the Creative Commons Attribution 4.0 International License.
Mouhssin Oufir, Kirsten Gillingwater, Matthias Hamburger, Isabel Roditi, Reto Brun, Pascal Mäser, Tanja Wenzler

Pharmaceutical Sciences, University of Basel; Medical Parasitology and Infection Biology, Swiss Tropical and Public Health Institute, Faculty of Science, University of Basel; Institute of Cell Biology, University of Bern

\begin{abstract}
African sleeping sickness is a tropical disease caused by Trypanosoma brucei gambiense or $T$. $b$. rhodesiense. Both subspecies are transmitted by the tsetse fly. In general, an infection is lethal without an effective treatment. We used a rodent efficacy model to test 4 compounds that had been previously identified in a novel in vitro screen as activators of parasite differentiation from bloodstream towards procyclic forms. The 4 compounds were trypanocidal in vitro. However, none of the compounds showed trypanocidal activity in vivo. Snapshot pharmacokinetic (PK) profiles indicated that the compound exposure was too low after intraperitoneal administration, which explains the lack of efficacy.
\end{abstract}

\section{Introduction}

Human African trypanosomiasis (HAT), also known as sleeping sickness, is a disease caused by 2 subspecies of T. brucei in sub-Saharan Africa. There is no vaccine available, and it is highly unlikely that one will be developed owing to frequent alterations of the parasites' variant surface glycoprotein (VSG) coat, a phenomenon known as antigenic variation [1]. The few drugs that are available to treat the disease all have serious drawbacks, but without treatment the disease is generally lethal [2].

T. brucei spp. are unicellular parasites. In the course of their life cycle in the mammalian host and the fly, the parasites develop into different life-cycle stages to adapt to their different environments. In this process they undergo changes in their morphology, gene expression, metabolism and surface coat. Bloodstream form trypanosomes are covered by a VSG coat that protects them from lysis by serum components. When the parasites are transferred to the tsetse midgut following a blood meal on an infected host, they differentiate into procyclic forms. During this process, the parasites start to shed the VSG coat that protects them in the mammal. The parasites simultaneously start to express procyclin proteins which form their coat in the fly midgut. Trypanosomes without a VSG coat are killed immediately when they are exposed to blood [3].

\section{Objective}

A recent publication presented twenty eight compounds that triggered the differentiation of bloodstream towards procyclic forms. All were trypanocidal in vitro [4]. We tested four of these compounds for their trypanocidal efficacy in a mouse model of trypanosome infection. 
Efficacy study with DIP-02, DIP-07, DIP-19.

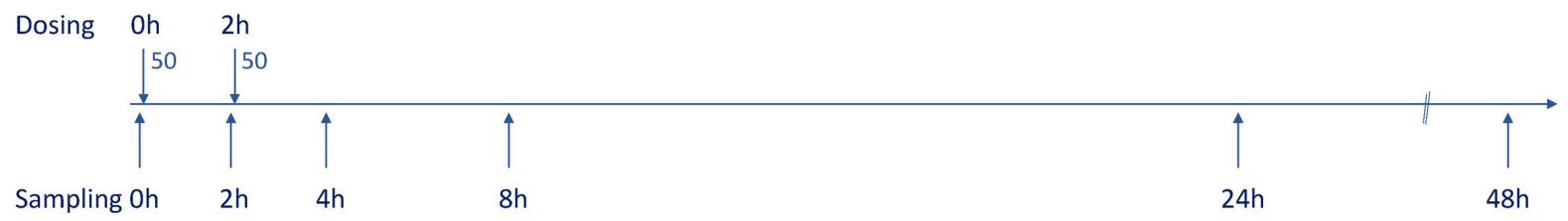

Efficacy study with DIP-03, DIP-07, DIP-19.

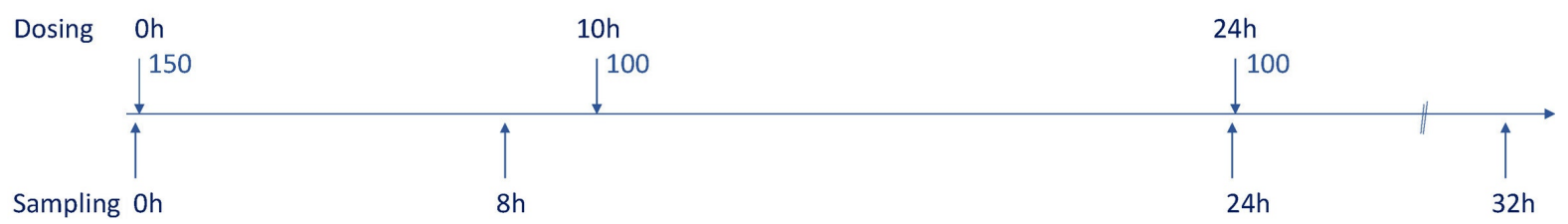

Efficacy study with DIP-03.

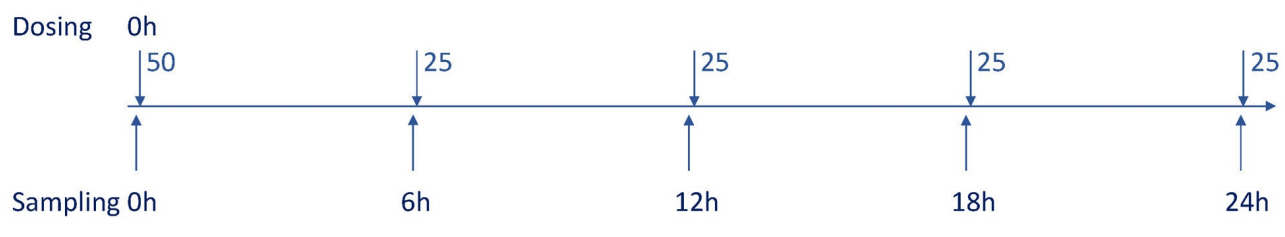



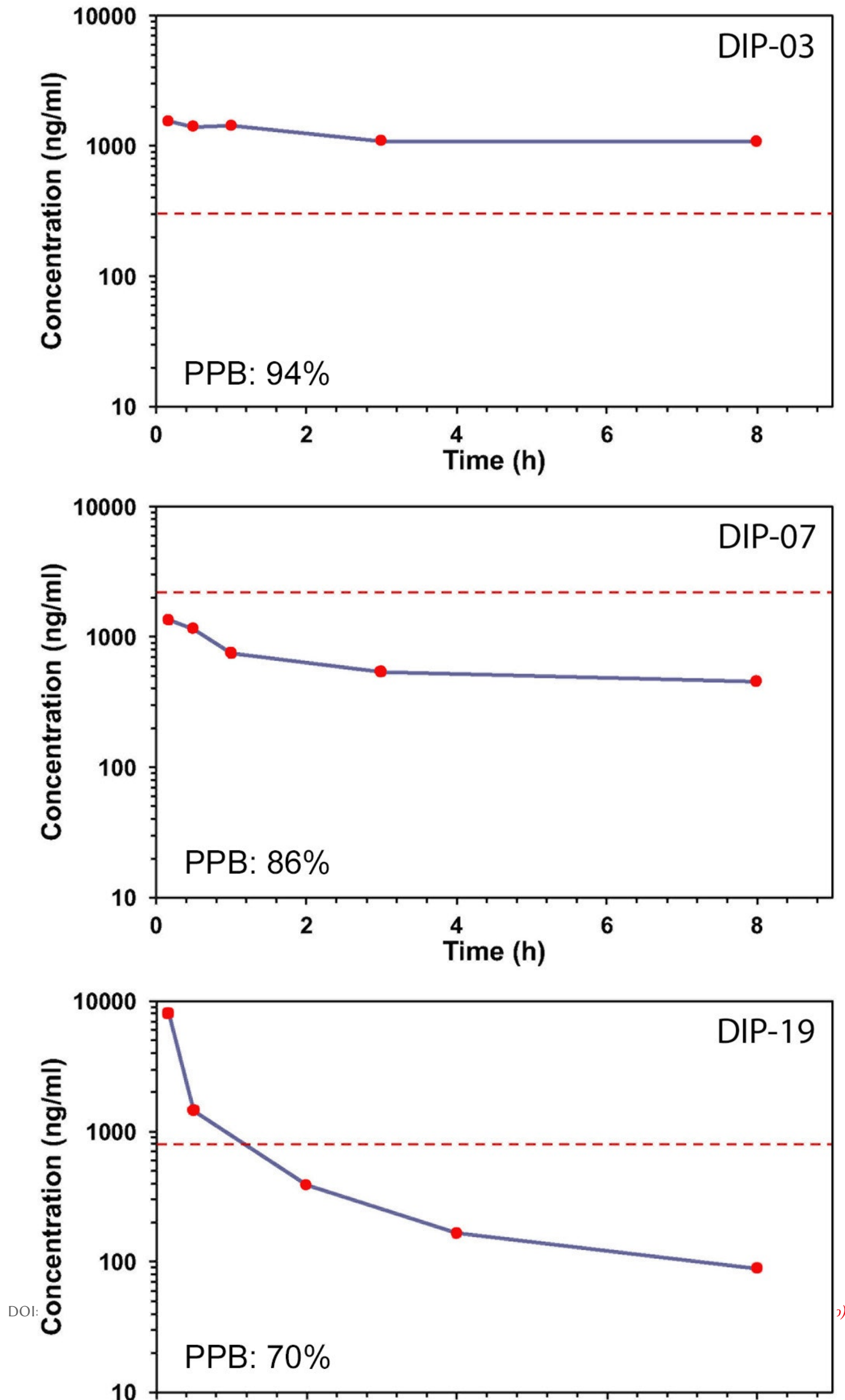
Figure Legend

Figure 1. Mouse model of T. b. rhodesiense infection (A) and pharmacokinetics (B).

(A) Drug injections and blood sampling times for parasitaemia for 3 independent efficacy studies in a mouse model of infection. Experiment 1 (upper panel): DIP-02, DIP-07 and DIP-19. Experiment 2 (middle panel): DIP-03, DIP-07 and DIP-19. Experiment 3 (lower panel): DIP-03.

(B) Snapshot pharmacokinetic studies over an $8 \mathrm{~h}$ period of DIP-03 at $50 \mathrm{mg} \mathrm{kg}^{-1}$, and DIP-07 and DIP-19 at $150 \mathrm{mg} \mathrm{kg}^{-1}$ i.p. The closed red circles show the drug levels in mouse plasma after a single dose. The red dotted lines indicate the $\mathrm{IC}_{50}$ concentration in a $40 \mathrm{~h}$ trypanosome viability assay ( $\mathrm{PPB}$, plasma protein binding).

\section{Results \& Discussion}

The four compounds DIP-02, DIP-03, DIP-07 and DIP-19 were initially selected based on their in vitro efficacy against T. brucei ( $\mathrm{IC}_{50}$ values of $1.0 \mu \mathrm{g} \mathrm{ml}^{-1}$ for DIP-o2; $0.30 \mu \mathrm{g} \mathrm{ml}^{-1}$ for DIP-03; $2.2 \mu \mathrm{g} \mathrm{ml}^{-1}$ for DIP-07; $0.80 \mu \mathrm{g} \mathrm{ml}^{-1}$ for DIP-19) and their in vitro activity in the GUS differentiation assay (GUS active at $\leq 2.5 \mu \mathrm{g} / \mathrm{ml}$ ) [4]. The GUS differentiation assay used a transgenic line that expresses $B$-glucuronidase under the control of a procyclin promoter [5]. Mice were infected with T. b. rhodesiense one day before the start of treatment and the effect of the treatments on blood parasite load was assessed qualitatively by microscopic examination of tail blood. The dosing regimens are described in figure $1 \mathrm{~A}$. The group size was limited to two mice per compound. The doses used in the first experiment were $2 \times 50 \mathrm{mg} \mathrm{kg}^{-1}$ i.p. at $2 \mathrm{~h}$ intervals for DIP-02, $-07,-19$ as we hypothesised that a short trigger might be sufficient to initiate the differentiation process (Fig. 1A, upper panel). At this dose, none of the compounds were active against trypanosomes. In addition, DIP-02 was toxic when administered at a total dose of 100 $\mathrm{mg} \mathrm{kg}^{-1}$ i.p. to infected mice.

In the second experiment with DIP-03, - 07 and -19, we started with a loading dose of 150 $\mathrm{mg} \mathrm{kg}^{-1}$ followed by further doses of $100 \mathrm{mg} \mathrm{kg}^{-1} 10 \mathrm{~h}$ and $24 \mathrm{~h}$ later (Fig. $1 \mathrm{~A}$, middle panel). DIP-03 was toxic at this high dose; DIP-07 and DIP-19 were tolerated at this dosage scheme, but did not reduce the parasitaemic load in tail blood.

In the third experiment, we tested DIP-03 at a loading dose of $50 \mathrm{mg} \mathrm{kg}^{-1}$ followed by four doses of $25 \mathrm{mg} \mathrm{kg}^{-1}$ spaced by intervals of $6 \mathrm{~h}$ (Fig. 1A, lower panel). Also with this dosing scheme we did not observe a significant parasite reduction in tail blood.

To determine whether the lack of efficacy was due to a poor compound exposure, we performed "snapshot" pharmacokinetic (PK) studies (Fig. 1B). This follows a simplified protocol that requires fewer mice and resources than a full PK [6] [7]. Although less informative compared to a full PK study, it provides sufficient information to determine whether the PK properties are likely to be the reason for treatment failure, and can also provide data to design a better treatment regime. DIP-o2 was excluded from the PK experiment due to the toxicity observed in the preliminary efficacy test. DIP-03 was tested at $50 \mathrm{mg} \mathrm{kg}^{-1}$ whereas DIP-07 and DIP-19 were administered at $150 \mathrm{mg} \mathrm{kg}^{-1}$ because the latter two compounds were tolerated at higher doses. Compounds were administered as a single i.p. dose and blood samples taken over a period of $8 \mathrm{~h}$ (Fig. 1B). Concentrations were measured in lithium heparin plasma samples analyzed by UPLC MS/MS as described in supplementary information. The results are summarized in supplementary table S2.

DIP-03 showed the best PK profile among the three compounds tested, with better exposure and a long half-life leading to a plasma drug concentration of $1.1 \mu \mathrm{g} \mathrm{ml}^{-1} 8 \mathrm{~h}$ after injection (Fig. 1B). The $\mathrm{C}_{\max }$ was reached $10 \mathrm{~min}$ after injection and the concentration was 5 -fold higher than the $\mathrm{IC}_{50}$ of the compound. However, the quantification method for the plasma samples did not distinguish between protein-bound and -unbound compound fractions. Plasma protein binding (PPB) data determined at $1 \mu \mathrm{M}$ in mouse plasma showed a high mean PPB of 94\% (Fig. 1B and Suppl. Table S2). High PPB was confirmed by serum-shift assays: increase from $10 \%$ to $40 \%$ inactivated horse serum in the in vitro viability assay ( $48 \mathrm{~h}$ Alamar blue assay) led to an 8 -fold increase of the $\mathrm{IC}_{50}$ value ( $\mathrm{p}<0.006$, two-tailed paired $\mathrm{t}$-test). The high PPB of DIP-03 indicates that the concentration of unbound drug in the mouse blood was too low in the efficacy experiments 
(at a dose reaching the toxicity limit) to kill the parasites in infected mice. The high PPB was most likely also the reason for the long half-life of DIP-03, given that a low free drug concentration significantly reduces clearance in an in vivo system [8].

The pharmacologically relevant distribution half-lives were short for DIP-o7 and DIP-19 (Fig. 1B). Additionally, the overall exposure of DIP-o7 was much too low depicted by a $\mathrm{C}_{\max }$ below the $\mathrm{IC}_{50}$ concentration of the compound. DIP-19 showed the shortest half-life but at the same time the highest $C_{\max }$ value $\left(8 \mu \mathrm{g} \mathrm{ml}^{-1}\right)$ which was 10 -fold higher than its $\mathrm{IC}_{50}$. However, with the short distribution half-life of DIP-19 in the plasma, dropping already after $30 \mathrm{~min}$ to $1.44 \mu \mathrm{g} \mathrm{ml}^{-1}$ that with a mean PPB of $70 \%$ resulted in a too low free drug concentration $\left(\mathrm{IC}_{50}=0.8 \mu \mathrm{g} \mathrm{ml}^{-1}\right.$ in the $40 \mathrm{~h}$ Alamar blue assay, Fig. 1B). Hence compound exposure of all three compounds administered by the i.p. route resulted in a too low free drug concentration that is too low to kill parasites in vivo.

\section{Conclusions}

Exploiting the complex life cycle of trypanosomes is a novel approach to chemotherapy. The hit compounds tested in vivo in this study were identified originally by targeting a parasite-specific process, in a screen for compounds that trigger parasite differentiation, and not by a typical phenotypic killing assay. However, the new compounds were not efficacious in vivo.

We evaluated three of the new compounds in a snapshot PK study and observed poor PK profiles with low exposure, short half-lives or, for DIP-03, high protein binding of $94 \%$ leading to insufficient free compound. As is often the case in drug discovery and development, a poor in vivo $\mathrm{PK}$ profile is a major obstacle for in vivo efficacy.

The hit compounds DIP-02, DIP-03, DIP-07 and DIP-19 could still serve as tool compounds to study the molecular mechanisms of parasite differentiation, but all four compounds have a negative therapeutic window and thus are poor drug candidates. In all likelihood they are not good leads for a drug discovery program for HAT.

\section{Limitations}

1. Drug solubility: A better formulation of the compounds could improve solubility and hence improve activity.

2. We hypothesised that a short trigger is sufficient to initiate the differentiation process. Therefore, the treatment regimen was rather short and with frequent doses to achieve a high $\mathrm{C}_{\max }$ or high initial plasma levels that would trigger the process. However, this hypothesis has not been validated yet.

3. The in vitro time to kill was short for the four compounds [4], suggesting that high and short drug exposure would be sufficient to kill the parasites. This might be different in vivo.

\section{Additional Information}

Methods and Supplementary Material

Please see https://sciencematters.io/articles/201807000009.

\section{Funding Statement}

This work was supported by the Bill and Melinda Gates Foundation through the Grand Challenges Explorations program, grant number 53289, and the Novartis Foundation for medical-biological Research, grant number 16B136.

\section{Ethics Statement}

All experiments with mice were performed in accordance with the guidelines of the Swiss authorities for animal welfare. All protocols and procedures used in the current study were reviewed and approved by the local veterinary authorities of the Canton Basel-Stadt, Switzerland (Permit numbers 2813 and 2566). 


\section{Citations}

[1] Monica R. Mugnier, C. Erec Stebbins, and F. Nina Papavasiliou. "Masters of Disguise: Antigenic Variation and the VSG Coat in Trypanosoma brucei”. In: PLOS Pathogens 12.9 (2016), e1005784. DoI: $10.1371 /$ journal ppat. 1005784 . urL: https : //doi.org/10.1371/journal.ppat. 1005784.

[2] Philippe Büscher et al. "Human African trypanosomiasis". In: The Lancet 390.10110 (2017), pp. 2397-2409. DOI: $10.1016 / \mathrm{s} 0140-6736$ (17) 31510-6. uRL: https : //doi.org/10.1016/s0140-6736(17)31510-6.

[3] A. FERRANTE and A.C. ALLISON. "Alternative pathway activation of complement by African trypanosomes lacking a glycoprotein coat”. In: Parasite Immunology 5.5 (1983), pp. 491-498. DOI:

10.1111/j.1365-3024.1983.tb00763.x. URL: https://doi.org/10.1111/j.13653024.1983.tb00763.x.

[4] Tanja Wenzler et al. "A new approach to chemotherapy: drug-induced differentiation kills African trypanosomes". In: Scientific Reports 6.1 (2016), p. 22451. DOI: 10.1038/srep22451. URL: https://doi.org/10.1038/srep22451.

[5] Sandro Sbicego et al. "The use of transgenic Trypanosoma brucei to identify compounds inducing the differentiation of bloodstream forms to procyclic forms". In: Molecular and Biochemical Parasitology 104.2 (1999), pp. 311-322. DOI: 10.1016/s0166-6851 (99)00157-7. URL: https : //doi.org/10.1016/s0166-6851(99)00157-7.

[6] Bo Liu et al. "Snapshot PK: a rapid rodent in vivo preclinical screening approach". In: Drug Discovery Today 13.7-8 (2008), pp. 36o-367. Dor: $10.1016 / \mathrm{j}$. drudis. 2007.10 .014$.
URL: https :

//doi.org/10.1016/j.drudis.2007.10.014.

[7] Chun Li et al. "A modern in vivo pharmacokinetic paradigm: combining snapshot, rapid and full PK approaches to optimize and expedite early drug discovery”. In: Drug Discovery Today 18.1-2 (2013), pp. 71-78. DOI:

10.1016/j.drudis. 2012.09.004. uRL: https: //doi.org/10.1016/j.drudis.2012.09.004

[8] Dennis A. Smith, Li Di, and Edward H. Kerns. "The effect of plasma protein binding on in vivo efficacy: misconceptions in drug discovery”. In: Nature Reviews Drug Discovery 9.12 (2010), pp. 929-939. DOI: $10.1038 / \mathrm{nrd3287}$. URL: https://doi.org/10.1038/nrd3287.

[9] Ihor Zahanich et al. "Phenoxymethyl 1,3-oxazoles and 1,2,4-oxadiazoles as potent and selective agonists of free fatty acid receptor 1 (GPR4o)". In: Bioorganic and Medicinal Chemistry Letters 25.16 (2015), pp. 3105-3111. DOI: 10.1016/j.bmc1.2015.06.018. URL: https: //doi.org/10.1016/j.bmc1.2015.06.018.

[10] Michael J. Banker, Tracey H. Clark, and John A. Williams. "Development and validation of a 96-well equilibrium dialysis apparatus for measuring plasma protein binding”. In: Journal of Pharmaceutical Sciences 92.5 (2003), pp. 967-974. DOI: 10.1002/jps. 10332. URL: https://doi.org/10.1002/jps. 10332

[11] Nigel J. Waters et al. "Validation of a Rapid Equilibrium Dialysis Approach for the Measurement of Plasma Protein Binding”. In: Journal of Pharmaceutical Sciences 97.10 (2008), pp. 4586-4595. DOI: $10.1002 / \mathrm{jps} .21317$. URL: https://doi.org/10.1002/jps. 21317. 